\title{
ASYMPTOTIC BEHAVIOUR OF SECOND-ORDER DIFFERENCE EQUATIONS
}

\author{
STEVO STEVIĆ 1
}

(Received 19 December, 2001; revised 19 September, 2003)

\begin{abstract}
In this paper we prove several growth theorems for second-order difference equations.
\end{abstract}

\section{Introduction}

In this paper we study second-order nonlinear difference equations of the form

$$
\Delta\left(c_{n-1} \Delta x_{n-1}\right)=d_{n} f\left(x_{n}\right)+g_{n}, \quad n \in \mathbf{N},
$$

where $x_{n}$ is the desired solution, and $c_{n}, d_{n}$ and $g_{n}$ are given real sequences.

In Section 2 we give and cite several auxiliary results which we shall apply in the sections which follow.

In Section 3 we study the second-order linear difference equation

$$
c_{n} x_{n+1}-b_{n} x_{n}+c_{n-1} x_{n-1}=0, \quad n \in \mathbf{N},
$$

where $x_{n}$ is the desired solution, and $b_{n}$ and $c_{n}$ are given real sequences. We investigate the asymptotic behaviour of the solutions of that equation under some conditions. We were motivated by [12] and [18].

This equation models, for example, the amplitude of oscillation of the weights on a discretely weighted vibrating string [2, p. 15-17].

A presentation of the results on similar problems for second-order differential equations can be found in [4].

In Section 4 we study the asymptotic behaviour of the second-order nonlinear difference equation (1.1).

\footnotetext{
${ }^{1}$ Mathematical Institute of Serbian Academy of Science, Knez Mihailova 35/I, 11000 Beograd, Serbia; e-mail: sstevic@ptt.yu and sstevo@matf.bg.ac.yu.

(C) Australian Mathematical Society 2004, Serial-fee code 1446-1811/04
} 


\section{Auxiliary results}

For an investigation into the asymptotic behaviour of the solution $x_{n}$, we need a few auxiliary lemmas. The first one is a discrete variant of the Bellman-Gronwall lemma. The continuous case of this lemma can be found in $[3,4]$ and [10]. Applications and further generalisations of this lemma can be found, for example, in [7,11-13,16$18,20]$.

LEMMA 2.1 ([16, p. 112]). If $x_{n}, b_{n}, c_{n} \geq 0$, and

$$
x_{n} \leq a_{n}+b_{n} \sum_{i=1}^{n-1} c_{i} x_{i}, \quad n \in \mathbf{N}
$$

then

$$
x_{n} \leq a_{n}+b_{n} \sum_{i=1}^{n-1} a_{i} c_{i} e^{\sum_{i=i+1}^{n-1} b_{j} c_{j}}, \quad n \in \mathbf{N}
$$

COROLLARY 2.2. If $x_{n}, c_{n} \geq 0, c$ is a positive constant, and

$$
x_{n} \leq c+\sum_{i=1}^{n-1} c_{i} x_{i}, \quad n \in \mathbf{N},
$$

then

$$
x_{n} \leq c \exp \left(\sum_{i=1}^{n-1} c_{i}\right), \quad n \in \mathbf{N} .
$$

Proof. By Lemma 2.1 we have

$$
x_{n} \leq c+c \sum_{i=1}^{n-1} c_{i} e^{\sum_{j=i+1}^{n-1} c_{j}}, \quad n \in \mathbf{N} .
$$

Applying the well-known inequality $x \leq e^{x}-1, x \geq 0$, we obtain

$$
\begin{aligned}
x_{n} & \leq c\left(1+\sum_{i=1}^{n-1}\left(e^{c_{i}}-1\right) e^{\sum_{j=i+1}^{n-1} c_{j}}\right) \\
& =c\left(1+\sum_{i=1}^{n-1}\left(e^{\sum_{j=i}^{n-1} c_{j}}-e^{\sum_{j=i+1}^{n-1} c_{j}}\right)\right)=c \exp \left(\sum_{i=1}^{n-1} c_{i}\right)
\end{aligned}
$$

as desired.

The following lemma was proved in [20]. 
LEMMA 2.3. If $x_{n}, c_{n} \geq 0$, $c$ is a positive constant, $p \in[0,1)$ and

$$
x_{n} \leq c+\sum_{i=1}^{n-1} c_{i} x_{i}^{p}, \quad n \in \mathbf{N},
$$

then

$$
x_{n} \leq\left(c^{1-p}+(1-p) \sum_{i=1}^{n-1} c_{i}\right)^{1 /(1-p)}, \quad n \in \mathbf{N} .
$$

The following lemma is a variant of the discrete version of Bihari's inequality [5]. This lemma generalises a discrete inequality in [11], see also [16, p. 114].

LEMMA 2.4. Assume that $x_{n}, a_{n}, b_{n}$ and $c_{n}$ are positive sequences, and that $a_{n}$ and $b_{n}$ satisfy the conditions

$$
1 \leq \frac{a_{n+1}}{a_{n}} \leq M, \quad 1 \leq \frac{b_{n+1}}{b_{n}} \leq M, \quad n \in \mathbf{N},
$$

and

$$
x_{n} \leq a_{n}+b_{n} \sum_{i=1}^{n-1} c_{i} g\left(x_{i}\right), \quad n \in \mathbf{N},
$$

where the real function $g(x)$ is continuous, nondecreasing and $g(x) \geq x$, for $x>0$. Then

$$
x_{n} \leq G^{-1}\left(G\left(a_{1}\right)+M \ln \frac{a_{n} b_{n}}{a_{1} b_{1}}+\sum_{i=1}^{n-1} b_{i+1} c_{i}\right), \quad n \in \overline{1, n_{0}},
$$

where $G(u)=\int_{\varepsilon}^{u}(d s / g(s))$ and

$$
n_{0}=\sup \left\{j \mid G\left(a_{1}\right)+M \ln \frac{a_{j} b_{j}}{a_{1} b_{1}}+\sum_{i=1}^{j-1} b_{i+1} c_{i} \in G\left(\mathbf{R}_{+}\right)\right\} .
$$

PROOF. Let $R_{n}=b_{n} \sum_{i=1}^{n-1} c_{i} g\left(x_{i}\right), s_{n}=\sum_{i=1}^{n} c_{i} g\left(x_{i}\right)$ and $v_{n}=R_{n}+a_{n}$. We can write (2.2) in the following form: $x_{n} \leq a_{n}+R_{n}, n \in \mathbf{N}$. From that we get

$$
\begin{aligned}
0 \leq v_{n+1}-v_{n} & =b_{n+1}\left(s_{n-1}+c_{n} g\left(x_{n}\right)\right)-b_{n} s_{n-1}+a_{n+1}-a_{n} \\
& =\left(b_{n+1}-b_{n}\right) s_{n-1}+b_{n+1} c_{n} g\left(x_{n}\right)+a_{n+1}-a_{n} \\
& =\frac{b_{n+1}-b_{n}}{b_{n}} R_{n}+b_{n+1} c_{n} g\left(x_{n}\right)+a_{n+1}-a_{n} .
\end{aligned}
$$

By the mean value theorem we have

$$
G\left(v_{n+1}\right)-G\left(v_{n}\right)=\left(v_{n+1}-v_{n}\right) / g\left(\zeta_{n}\right),
$$


for some $\zeta_{n} \in\left(v_{n}, v_{n+1}\right)$. From (2.4) and (2.5) we obtain

$$
\begin{aligned}
G\left(v_{n+1}\right)-G\left(v_{n}\right) & =\frac{1}{g\left(\zeta_{n}\right)}\left(\frac{b_{n+1}-b_{n}}{b_{n}} R_{n}+b_{n+1} c_{n} g\left(x_{n}\right)+a_{n+1}-a_{n}\right) \\
& \leq \frac{b_{n+1}-b_{n}}{b_{n}}+b_{n+1} c_{n}+\frac{a_{n+1}-a_{n}}{g\left(a_{n}\right)},
\end{aligned}
$$

since $g\left(a_{n}\right) \leq g\left(v_{n}\right) \leq g\left(\zeta_{n}\right)$.

Summing (2.6) from 1 to $n-1$, we obtain

$$
G\left(v_{n}\right) \leq G\left(a_{1}\right)+\sum_{i=1}^{n-1} \frac{b_{i+1}-b_{i}}{b_{i}}+\sum_{i=1}^{n-1} b_{i+1} c_{i}+\sum_{i=1}^{n-1} \frac{a_{i+1}-a_{i}}{g\left(a_{i}\right)} .
$$

From the conditions of the theorem we get

$$
G\left(v_{n}\right) \leq G\left(a_{1}\right)+M \sum_{i=1}^{n-1} \frac{b_{i+1}-b_{i}}{b_{i+1}}+\sum_{i=1}^{n-1} b_{i+1} c_{i}+M \sum_{i=1}^{n-1} \frac{a_{i+1}-a_{i}}{a_{i+1}} .
$$

Since every positive nondecreasing sequence $y_{n}$ satisfies the following inequality:

$$
\sum_{i=1}^{n-1} \frac{y_{i+1}-y_{i}}{y_{i+1}} \leq \int_{y_{1}}^{y_{n}} \frac{d t}{t}=\ln \frac{y_{n}}{y_{1}}
$$

we obtain

$$
G\left(v_{n}\right) \leq G\left(a_{1}\right)+M \ln \frac{a_{n} b_{n}}{a_{1} b_{1}}+\sum_{i=1}^{n-1} b_{i+1} c_{i},
$$

and so (2.3) follows.

LEMMA $2.5\left(\left[14\right.\right.$, p. 281]). Let $v_{n}>0$ and assume that the series $\sum_{i=1}^{+\infty} u_{n}$ and $\sum_{i=1}^{+\infty} v_{n}$ converge. Then

$$
\lim _{n \rightarrow+\infty} \frac{u_{n}}{v_{n}}=c \quad \Rightarrow \quad \lim _{n \rightarrow+\infty} \frac{\sum_{i=n}^{+\infty} u_{i}}{\sum_{i=n}^{+\infty} v_{i}}=c
$$

\section{The linear equation case}

We are now in a position to formulate and to prove the main results in the case of a linear equation. In what follows we exclude the trivial solution from consideration.

Observe that the difference equation $x_{n+1}-2 x_{n}+x_{n-1}=0$ has a general solution in the form $x_{n}=a n+b$ for some $a, b \in \mathbf{R}$. In the following theorem we give 
one sufficient condition, such that the difference equation (1.2) has solutions which approach those of $x_{n+1}-2 x_{n}+x_{n-1}=0$. An equivalent result was proved in [6, p. 377]. We present here a different proof which follows the lines of the proof given in [4] in the continuous case. The proof essentially appears in [9, Theorem 7.17] but contains a gap. Hence we present here a correct proof.

THEOREM 3.1. Consider (1.2) where $\sum_{i=1}^{+\infty} i\left(\left|1-c_{i}\right|+\left|2-b_{i}\right|\right)<+\infty$. Then the general solution is asymptotic to an $+b$ as $n \rightarrow \infty$, where a or b may be zero, but not both simultaneously.

Proof. Without loss of generality we may suppose $c_{n}>0, n \in \mathbf{N} \cup\{0\}$. Let us write (1.2) in the following form:

$$
\Delta\left(c_{n-1} \Delta x_{n-1}\right)=d_{n} x_{n}
$$

It is clear that $d_{n}=b_{n}-c_{n}-c_{n-1}$. Let $y_{n}=x_{n+1}-x_{n}$. Then from (3.1) we have

$$
c_{n} y_{n}-c_{n-1} y_{n-1}=d_{n} x_{n}, \quad n \in \mathbf{N} .
$$

Summing (3.2) from 1 to $n-1$, we obtain

$$
x_{n}-x_{n-1}=\frac{1}{c_{n-1}}\left(c_{0} y_{0}+\sum_{i=1}^{n-1} d_{i} x_{i}\right) .
$$

Now, summing (3.3) from 1 to $n$, we get

$$
x_{n}=x_{0}+c_{0} y_{0} \sum_{i=1}^{n} \frac{1}{c_{i-1}}+\sum_{i=1}^{n} \frac{1}{c_{i-1}}\left(\sum_{j=1}^{i-1} d_{j} x_{j}\right) .
$$

By the condition of the theorem, $c_{n} \rightarrow 1$ as $n \rightarrow \infty$. Therefore

$$
\lim _{n \rightarrow \infty} \frac{1}{n} \sum_{i=1}^{n} \frac{1}{c_{i-1}}=1
$$

and the sequences $\left(\left|1 / c_{n}\right|\right)$ and $\left(\left|(1 / n) \sum_{i=1}^{n} 1 / c_{i-1}\right|\right)$ are bounded, for example, by $M>0$. 
It follows that

$$
\begin{aligned}
\frac{\left|x_{n}\right|}{n} & \leq \frac{\left|x_{0}\right|}{n}+\left|c_{0}\right|\left|x_{1}-x_{0}\right| M+\frac{M}{n} \sum_{i=1}^{n} \sum_{j=1}^{i-1}\left|d_{j}\right|\left|x_{j}\right| \\
& =\frac{\left|x_{0}\right|}{n}+\left|c_{0}\right|\left|x_{1}-x_{0}\right| M+\frac{M}{n} \sum_{i=1}^{n}(n-i)\left|d_{i}\right|\left|x_{i}\right| \\
& \leq\left|x_{0}\right|+\left|c_{0}\right|\left|x_{1}-x_{0}\right| M+M \sum_{i=1}^{n-1} i\left|d_{i}\right| \frac{\left|x_{i}\right|}{i} \\
& \leq\left(\left|x_{0}\right|+\left|c_{0}\right|\left|x_{1}-x_{0}\right| M\right) \exp \left(M \sum_{i=1}^{n-1} i\left|d_{i}\right|\right) \\
& \leq\left(\left|x_{0}\right|+\left|c_{0}\right|\left|x_{1}-x_{0}\right| M\right) \exp \left(M\left|1-c_{0}\right|+3 M \sum_{i=1}^{\infty} i\left(\left|1-c_{i}\right|+\left|b_{i}-2\right|\right)\right) \\
& =M_{1}<\infty
\end{aligned}
$$

where in the third inequality we applied Corollary 2.2 .

From (3.4) we obtain

$$
\sum_{i=1}^{n-1}\left|d_{i}\right|\left|x_{i}\right| \leq M_{1} \sum_{i=1}^{n-1} i\left|d_{i}\right| \leq M_{1} \sum_{i=1}^{+\infty} i\left|d_{i}\right|<\infty
$$

By (3.3), we can conclude that there exists $\lim _{n \rightarrow \infty}\left(x_{n}-x_{n-1}\right)=a$. If this limit is not zero, we have $x_{n} \sim$ an as $n \rightarrow \infty$. In particular, $x_{n} \neq 0$ for sufficiently large $n$. To ensure that $\lim _{n \rightarrow \infty}\left(x_{n}-x_{n-1}\right)$ is not zero, we may choose $x_{1}$ and $x_{0}$ such that

$$
\left|c_{0}\right|\left|x_{1}-x_{0}\right|-M_{1} \sum_{i=1}^{+\infty} i\left|d_{i}\right|>0
$$

Further, we shall use the fact that

$$
z_{n}=x_{n}\left(C+\sum_{i=1}^{n-1} \prod_{j=1}^{i-1} \frac{c_{j} x_{j}}{c_{j+1} x_{j+2}}\right)=x_{n}\left(C+x_{1} x_{2} c_{1} \sum_{i=1}^{n-1} \frac{1}{c_{i} x_{i} x_{i+1}}\right)
$$

is another solution, linearly independent of $x_{n}$; see, for example, [15, p. 160]. Therefore

$$
z_{n}=x_{n}\left(\sum_{i=n}^{+\infty} \frac{1}{c_{i} x_{i} x_{i+1}}\right)
$$


is another solution, linearly independent of $x_{n}$. It is well-defined since $x_{n} \neq 0$ for sufficiently large $n$ and $x_{n} \sim a n$ as $n \rightarrow \infty$. By Lemma 2.5 , we obtain

$$
\lim _{n \rightarrow \infty} z_{n}=\lim _{n \rightarrow \infty} \frac{x_{n}}{n} \lim _{n \rightarrow \infty} \frac{\left(\sum_{i=n}^{+\infty} 1 /\left(c_{i} x_{i} x_{i+1}\right)\right)}{1 / n}=a \lim _{n \rightarrow \infty} \frac{1 /\left(c_{n} x_{n} x_{n+1}\right)}{1 /(n(n+1))}=\frac{1}{a} .
$$

Thus the solution $a z_{n}$ is asymptotic to 1 as $n \rightarrow \infty$, and therefore every solution of our difference equation is asymptotic to $a n+b$ as $n \rightarrow \infty$.

If $y_{n}$ is an arbitrary solution of (1.2) and if $\lim _{n \rightarrow+\infty} y_{n}$ is finite, then $y_{n}=c z_{n}$, $n \in \mathbf{N}$ for some $c \in \mathbf{R}$. Thus if $\lim _{n \rightarrow+\infty} y_{n}=0$ we obtain $c=0$, that is, $y_{n}$ is a trivial solution. In the other cases $\lim _{n \rightarrow+\infty} y_{n}=\infty$ and so $a \neq 0$.

Before formulating the following result we would like to point out that recently W. Trench investigated principal and nonprincipal solutions of the nonoscillatory equation (3.1) in [19].

Let us investigate what happens in the case of $\sum_{i=1}^{+\infty} i\left|d_{i}\right|=+\infty$. The simplest case is when $d_{n}=1 / n^{\alpha}, \alpha \in(0,2]$ and $c_{n}=1$ for all $n \in \mathbf{N}$.

THEOREM 3.2. Consider the equation

$$
x_{n+1}-2 x_{n}+x_{n-1}=d_{n} x_{n},
$$

where $d_{i}=c / i^{\alpha}, i \in \mathbf{N}, c \in \mathbf{R}, \alpha \in(0,2]$. Then for every solution of (3.6) the asymptotic formula

(1) $x_{n}=\mathscr{O}\left(n^{|c|+1}\right)$, for $\alpha=2$,

(2) $x_{n}=\mathscr{O}\left(n e^{|c| n^{2-\alpha} /(2-\alpha)}\right)$, for $\alpha \in(0,2)$

holds.

PROOF. Let $y_{n}=x_{n+1}-x_{n}$. As in Theorem 3.1 we have

$$
x_{n}-x_{n-1}=y_{n-1}=y_{0}+c \sum_{i=1}^{n-1} \frac{1}{i^{\alpha}} x_{i} .
$$

Now, summing (3.7) from 1 to $n$, and by a simple calculation we obtain

$$
x_{n}=x_{0}+n\left(x_{1}-x_{0}\right)+c \sum_{i=1}^{n-1}(n-i) \frac{1}{i^{\alpha}} x_{i} .
$$

It follows that

$$
\left|x_{n}\right| \leq\left|x_{0}\right|+n\left|x_{1}-x_{0}\right|+n|c| \sum_{i=1}^{n-1} \frac{1}{i^{\alpha}}\left|x_{i}\right|
$$


and further

$$
\begin{aligned}
\frac{\left|x_{n}\right|}{n} & \leq \frac{\left|x_{0}\right|}{n}+\left|x_{1}-x_{0}\right|+|c| \sum_{i=1}^{n-1} \frac{1}{i^{\alpha}}\left|x_{i}\right| \\
& \leq\left|x_{0}\right|+\left|x_{1}-x_{0}\right|+|c| \sum_{i=1}^{n-1} \frac{1}{i^{\alpha-1}} \frac{\left|x_{i}\right|}{i} .
\end{aligned}
$$

Applying the discrete Bellman-Gronwall lemma, we obtain

$$
\frac{\left|x_{n}\right|}{n} \leq\left(\left|x_{0}\right|+\left|x_{1}-x_{0}\right|\right) \exp \left(|c| \sum_{i=1}^{n-1} \frac{1}{i^{\alpha-1}}\right)
$$

We have

$$
\sum_{i=1}^{n-1} \frac{1}{i^{\alpha-1}} \leq \begin{cases}1+\int_{1}^{n-1} \frac{d t}{t^{\alpha-1}}, & \text { for } \alpha \in(1,2] \\ \int_{1}^{n} \frac{d t}{t^{\alpha-1}}, & \text { for } \alpha \in(0,1] .\end{cases}
$$

Thus we have

$$
\begin{aligned}
& \sum_{i=1}^{n-1} \frac{1}{i} \leq 1+\ln (n-1), \\
& \sum_{i=1}^{n-1} \frac{1}{i^{\alpha-1}} \leq \begin{cases}1+\frac{(n-1)^{2-\alpha}-1}{2-\alpha}, & \text { for } \alpha \in(1,2), \\
\int_{1}^{n} t^{1-\alpha} d t=\frac{n^{2-\alpha}-1}{2-\alpha}, & \text { for } \alpha \in(0,1] .\end{cases}
\end{aligned}
$$

From all of the above, the result follows.

EXAMPLE 1. Consider the difference equation

$$
x_{n+1}-2 x_{n}+x_{n-1}=\frac{2}{n^{2}} x_{n}, \quad n \geq 1 .
$$

This equation is derived from (3.6) by putting $c=2$. Its solution is $x_{n}=n^{2}$.

EXAMPLE 2. Consider the difference equation

$$
x_{n+1}-2 x_{n}+x_{n-1}=\frac{6}{n^{2}} x_{n}, \quad n \geq 1 .
$$

This equation is derived from (3.6) by putting $c=6$. Its solution is $x_{n}=n^{3}$. 
QUESTION 1. These two examples motivate us to conjecture that in Theorem 3.2

$$
x_{n}=\mathscr{O}\left(n^{(1+\sqrt{4 \mid c+1}) / 2}\right)
$$

holds. Is it really so and for what $c \in \mathbf{R}$ does this formula hold?

These examples show that for a fixed $\alpha$ the growth of the solution of (3.6) really depends on the parameter $c$.

THEOREM 3.3. There exists a sequence $d_{n}$ such that $\lim _{n \rightarrow+\infty} d_{n}=0, \sum_{i=1}^{+\infty} i\left|d_{i}\right|=$ $+\infty$ and for some solutions of (3.6), $n^{k} \prec\left|x_{n}\right|$ holds for every $k \in \mathbf{N}$.

Proof. Consider the equation

We know that

$$
x_{n+1}-2 x_{n}+x_{n-1}=\frac{1}{n^{\alpha}} x_{n}, \quad \alpha \in(0,2) .
$$

$$
x_{n}=x_{0}+n\left(x_{1}-x_{0}\right)+\sum_{i=1}^{n-1}(n-i) \frac{1}{i^{\alpha}} x_{i} .
$$

Let $x_{0}=0$ and $x_{1}=1$. It is easy to see that in that case $x_{n} \geq 0$ for every $n \in \mathbf{N}$. Thus we have $x_{n} \geq n$, for $n \in \mathbf{N}$. Applying this in (3.9) we obtain

$$
x_{n} \geq n+\sum_{i=1}^{n-1}(n-i) \frac{1}{i^{\alpha}} i=n+n \sum_{i=1}^{n-1} \frac{1}{i^{\alpha-1}}-\sum_{i=1}^{n-1} \frac{1}{i^{\alpha-2}} .
$$

Since

$$
\sum_{i=1}^{n-1} \frac{1}{i^{\beta}} \sim \int_{1}^{n-1} \frac{d t}{t^{\beta}} \sim \frac{n^{1-\beta}}{1-\beta}, \quad \text { for } \beta \neq 1
$$

we have that there is a $c_{1}>0$ such that

$$
x_{n} \geq n+c_{1} n^{3-\alpha} \geq c_{1} n^{3-\alpha},
$$

for all $n \in \mathbf{N}$. Hence $n^{\beta} \prec\left|x_{n}\right|$, for $\beta<3-\alpha$. Applying (3.10) in (3.9) we obtain

$$
x_{n} \geq n+c_{1} \sum_{i=1}^{n-1}(n-i) \frac{1}{i^{\alpha}} i^{3-\alpha}=n+n c_{1} \sum_{i=1}^{n-1} \frac{1}{i^{2 \alpha-3}}-c_{1} \sum_{i=1}^{n-1} \frac{1}{i^{2 \alpha-4}},
$$

from which it follows that there is a $c_{2}>0$ such that $x_{n} \geq n+c_{2} n^{5-2 \alpha}$, for all $n \in \mathbf{N}$.

Repeating the previous procedure and by induction we obtain that for every $k \in \mathbf{N}$, there is a constant $c_{k}>0$ such that

$$
x_{n} \geq n+c_{k} n^{2 k+1-k \alpha} \geq c_{k} n^{(2-\alpha) k+1},
$$

for every $n \in \mathbf{N}$. From this and since $\alpha \in(0,2)$ the result follows. 


\section{The nonlinear equation case}

In this section we shall study the asymptotic behaviour of the second-order nonlinear difference equation (1.1).

THEOREM 4.1. Consider (1.1) where

(a) $c_{n} \geq \delta>0, n \geq n_{0}$;

(b) $g_{n}$ is an arbitrary real sequence;

(c) $d_{n}$ is a real sequence such that $\sum_{i=1}^{+\infty} i\left|d_{i}\right|<+\infty$;

(d) $f$ is a real function such that $|f(x)| \leq L|x|^{\alpha}, x \in \mathbf{R}$, for some $L>0$ and some $\alpha \in[0,1]$.

Then the following asymptotic formula holds:

$$
x_{n}=\mathscr{O}\left(n+n \sum_{i=1}^{n-1}(n-i)\left|g_{i}\right|\right) \quad \text { as } n \rightarrow+\infty .
$$

PROOF. Let $y_{n}=c_{n}\left(x_{n+1}-x_{n}\right)$. Then from (1.1) we have

$$
y_{n}-y_{n-1}=d_{n} f\left(x_{n}\right)+g_{n}, \quad n \in \mathbf{N} .
$$

As in Theorem 3.1 we can obtain

$$
x_{n}=x_{0}+c_{0} y_{0} \sum_{i=1}^{n} \frac{1}{c_{i-1}}+\sum_{i=1}^{n} \frac{1}{c_{i-1}}\left(\sum_{j=1}^{i-1}\left(d_{j} f\left(x_{j}\right)+g_{j}\right)\right) .
$$

By conditions (a), (d) and some simple calculations, we obtain

$$
\begin{aligned}
\left|x_{n}\right| & \leq\left|x_{0}\right|+n\left|c_{0}\right|\left|x_{1}-x_{0}\right| M+M \sum_{i=1}^{n-1}(n-i)\left|g_{i}\right|+n M \sum_{i=1}^{n-1}\left|d_{i}\right|\left|f\left(x_{i}\right)\right| \\
& \leq\left|x_{0}\right|+n\left|c_{0}\right|\left|x_{1}-x_{0}\right| M+M \sum_{i=1}^{n-1}(n-i)\left|g_{i}\right|+n M L \sum_{i=1}^{n-1}\left|d_{i}\right|\left|x_{i}\right|^{\alpha},
\end{aligned}
$$

where $M$ is an upper bound for the sequence $\left(\left|1 / c_{n}\right|\right)$.

Let $A_{n}=\sum_{i=1}^{n-1}(n-i)\left|g_{i}\right|$. By the well-known inequality $|x|^{\alpha} \leq 1+|x|, x \in \mathbf{R}$, $\alpha \in[0,1]$, we have

$$
\left|x_{n}\right|+1 \leq c\left(1+n+A_{n}\right)+c n \sum_{i=1}^{n-1}\left|d_{i}\right|\left(\left|x_{i}\right|+1\right),
$$

for some $c \geq 1$. 
By Lemma 2.1 and condition (c), we obtain

$$
\begin{aligned}
\left|x_{n}\right|+1 & \leq c\left(1+n+A_{n}\right)+c^{2} n \sum_{i=1}^{n-1}\left(1+i+A_{i}\right)\left|d_{i}\right| e^{c \sum_{j=i+1}^{n-1} j\left|d_{j}\right|} \\
& \leq c\left(1+n+A_{n}\right)+c_{1} n \sum_{i=1}^{n-1}\left(1+i+A_{i}\right)\left|d_{i}\right|
\end{aligned}
$$

where $c_{1}=c^{2} e^{c \sum_{i=1}^{+\infty} i\left|d_{i}\right|}$.

From (4.2) we obtain

$$
\frac{\left|x_{n}\right|+1}{n+n A_{n}} \leq c(1 / n+1)+c_{1} \sum_{i=1}^{n-1}(1+i)\left|d_{i}\right|,
$$

since $A_{i}$ is nondecreasing. From (4.3), the result follows.

REMARK 1. If $\alpha>1$, then the theorem does not hold.

EXAMPLE 3. Consider the difference equation

$$
x_{n+1}-2 x_{n}+x_{n-1}=\frac{2}{n^{2 \alpha}} x_{n}^{\alpha}, \quad n \geq 1, \alpha>1 .
$$

This equation satisfies all conditions of Theorem 4.1 except $\alpha \in[0,1]$. For this equation $x_{n}=n^{2}$ is a solution, but $x_{n}$ is not $\mathscr{O}(n)$. Also $\lim _{n \rightarrow \infty}\left(x_{n+1}-x_{n}\right)$ is not finite.

EXAMPLE 4. Consider the difference equation

$$
x_{n+1}-2 x_{n}+x_{n-1}=\frac{6}{n^{3 \alpha-1}} x_{n}^{\alpha}, \quad n \geq 1, \alpha>1 .
$$

For this equation $x_{n}=n^{3}$ is a solution, but $x_{n}$ is not $\mathscr{O}(n)$.

THEOREM 4.2. Consider (1.1) where

(a) $c_{n} \geq \delta>0, n \geq n_{0}$;

(b) $g_{n}$ is a real sequence such that $\sum_{i=1}^{+\infty}\left|g_{i}\right|<+\infty$;

(c) $d_{n}$ is a real sequence such that $\sum_{i=1}^{+\infty} i^{\alpha}\left|d_{i}\right|<+\infty$, for some $\alpha \in[0,1)$;

(d) $f$ is a real function such that $|f(x)| \leq L|x|^{\alpha}, x \in \mathbf{R}$, for some $L>0$.

Then for every solution $x_{n}$ of (1.1), $x_{n}=\mathscr{O}(n)$ as $n \rightarrow+\infty$ and the following limit is finite:

$$
\lim _{n \rightarrow+\infty} c_{n-1}\left(x_{n}-x_{n-1}\right)
$$


Proof. As in the proof of Theorem 4.1 we have

$$
\begin{aligned}
\left|x_{n}\right| & \leq\left|x_{0}\right|+n\left|c_{0}\right|\left|x_{1}-x_{0}\right| M+M \sum_{i=1}^{n-1}(n-i)\left|g_{i}\right|+n M L \sum_{i=1}^{n-1}\left|d_{i}\right|\left|x_{i}\right|^{\alpha} \\
& \leq c\left(1+n+n \sum_{i=1}^{n-1}\left|g_{i}\right|\right)+c n \sum_{i=1}^{n-1}\left|d_{i}\right|\left|x_{i}\right|^{\alpha}
\end{aligned}
$$

for some $c>0$. Since $\sum_{i=1}^{+\infty}\left|g_{i}\right|<+\infty$, we have

$$
\frac{\left|x_{n}\right|}{n} \leq c_{1}+c \sum_{i=1}^{n-1}\left|d_{i}\right|\left|x_{i}\right|^{\alpha} \leq c_{1}+c \sum_{i=1}^{n-1} i^{\alpha}\left|d_{i}\right|\left(\frac{\left|x_{i}\right|}{i}\right)^{\alpha} .
$$

For $\alpha \in[0,1)$, by Lemma 2.3 we get

$$
\begin{aligned}
\frac{\left|x_{n}\right|}{n} & \leq\left(c_{1}^{1-\alpha}+(1-\alpha) c \sum_{i=1}^{n-1} i^{\alpha}\left|d_{i}\right|\right)^{1 /(1-\alpha)} \\
& \leq\left(c_{1}^{1-\alpha}+(1-\alpha) c \sum_{i=1}^{+\infty} i^{\alpha}\left|d_{i}\right|\right)^{1 /(1-\alpha)}<+\infty,
\end{aligned}
$$

thus the first part of the theorem follows.

From the above we know that there exists $M>0$ such that $\left|x_{n}\right| \leq M n$, for every $n \in \mathbf{N}$. Summing (4.1) from $n+1$ to $n+p$, we obtain

$$
y_{n+p}-y_{n}=\sum_{i=n+1}^{n+p} g_{i}+\sum_{i=n+1}^{n+p} d_{i} f\left(x_{i}\right) .
$$

Hence

$$
\begin{aligned}
\left|y_{n+p}-y_{n}\right| & \leq \sum_{i=n+1}^{n+p}\left|g_{i}\right|+\sum_{i=n+1}^{n+p}\left|d_{i}\right|\left|x_{i}\right|^{\alpha} \\
& \leq \sum_{i=n+1}^{n+p}\left|g_{i}\right|+M^{\alpha} \sum_{i=n+1}^{n+p} i^{\alpha}\left|d_{i}\right| .
\end{aligned}
$$

By the conditions of the theorem and Cauchy's criteria we obtain the result.

REMARK 2. Theorem 4.2 is a generalisation of the main result in [8]. The result also holds in the case $\alpha=1$ (see, for example, [1, Problem 6.24.40]). Using Corollary 2.2 instead of Lemma 2.3 in the above proof we can prove the theorem in this case.

REMARK 3. Example 3 shows that we cannot allow that $\sum_{i=1}^{+\infty} i^{\alpha}\left|d_{i}\right|=+\infty$, for some $\alpha \in[0,1)$. Indeed, in that case $d_{n}=2 / n^{2 \alpha}$ and $\sum_{i=1}^{+\infty} i^{\alpha}\left|d_{i}\right|=\sum_{i=1}^{+\infty} 2 / i^{\alpha}=$ $+\infty$, if $\alpha \in[0,1)$. On the other hand $x_{n}=n^{2}$ is a solution such that $x_{n} \neq \mathscr{O}(n)$. 
THEOREM 4.3. Consider (1.1), where $g_{n}=0$ and $c_{n}=1$ for all $n \in \mathbf{N}, f$ is a real even nondecreasing function for $x>0, f(x) \geq|x|$ for $x \in \mathbf{R}$ and $\int^{+\infty} d s / f(s)=$ $+\infty$. Then for every solution $x_{n}$ of $(1)$ we have

$$
x_{n}=\mathscr{O}\left(G^{-1}\left(G(2 c)+4 \ln n+\sum_{i=1}^{n-1}(i+1)\left|d_{i}\right|\right)\right) \text { as } n \rightarrow+\infty,
$$

where $c=\max \left\{1,\left|x_{0}\right|,\left|x_{0}-x_{1}\right|\right\}$ and $G(u)=\int_{\varepsilon}^{u} d s / f(s), \varepsilon \in(0,1)$.

PROOF. As in Theorem 4.1 we have

$$
\begin{aligned}
\left|x_{n}\right| & \leq\left|x_{0}\right|+n\left|x_{1}-x_{0}\right|\left|c_{0}\right|+n \sum_{i=1}^{n-1}\left|d_{i}\right|\left|f\left(x_{i}\right)\right| \\
& \leq c(1+n)+c n \sum_{i=1}^{n-1}\left|d_{i}\right| f\left(\left|x_{i}\right|\right) .
\end{aligned}
$$

By Lemma 2.4, we get

$$
\left|x_{n}\right| \leq G^{-1}\left(G(2 c)+2 \ln \frac{n(n+1)}{2}+\sum_{i=1}^{n-1}(i+1)\left|d_{i}\right|\right), \quad \text { for } n \in \mathbf{N}
$$

since $\int_{-\infty} d s / f(s)=\int^{+\infty} d s / f(s)=+\infty$, from which the result follows.

\section{References}

[1] R. P. Agarwal, Difference equations and inequalities, Pure Appl. Math. 228, 2nd ed. (Marcel Dekker, New York, 2000).

[2] F. V. Atkinson, Discrete and continuous boundary problems (Academic Press, New York, 1964).

[3] R. Bellman, "The stability of solutions of linear differential equations", Duke Math. J. 10 (1943) 643-647.

[4] R. Bellman, Stability theory of differential equations (McGraw-Hill, New York, 1953).

[5] I. Bihari, "A generalization of a lemma of Bellman and its application to uniqueness problems of differential equations", Acta Math. Acad. Sci. Hungar. 7 (1956) 81-94.

[6] T. S. Chihara and P. Nevai, "Orthogonal polynomials and measures with finitely many point masses", J. Approx. Theory 35 (1982) 370-380.

[7] F. S. De Blasi and J. Schinas, "On the stability of difference equations in Banach spaces", Analele stintificae ale Universitatii Al. Cuza, lasi Sectia la. Matematica. 20 (1974) 65-80.

[8] A. Drozdowicz, "On the asymptotic behavior of solutions of the second order difference equations", Glas. Mat. Ser. III 22 (42) (1987) 327-333.

[9] S. N. Elaydi, An introduction to difference equations (Springer, New York, 1996).

[10] T. Gronwall, "Note on the derivatives with respect to a parameter of the solutions of a system of differential equations", Ann. of Math. (2) 20 (1919) 292-296. 
[11] T. Hull and W. Luxemburg, "Numerical methods and existence theorems for ordinary differential equations", Numer. Math. 2 (1960) 30-41.

[12] E. Kurpinar and G. Sh. Guseinov, "The boundedness of solutions of second-order difference equations", Indian J. Math. 37 (1995) 113-122.

[13] A. Mate and P. Nevai, "Sublinear perturbations of the differential equation $y^{(n)}=0$ and of the analogous difference equation", J. Differential Equations 53 (1984) 234-257.

[14] D. S. Mitrinović and D. D. Adamović, Sequences and series (Naučna Knjiga, Beograd, 1990).

[15] D. S. Mitrinović and J. D. Kečkić, Methods for calculation of finite sums (Naučna Knjiga, Beograd, 1990).

[16] D. S. Mitrinović and J. E. Pečarić, Differential and integral inequalities (Naučna Knjiga, Beograd, 1988).

[17] C. Papaschinopoulos, "On the summable manifold for discrete systems", Math. Japonica 33 (1988) $457-468$.

[18] S. Stevic, "Growth theorems for homogeneous second-order difference equations", ANZIAM J. 43 (2002) 559-566.

[19] W. F. Trench, "Linear perturbations of a nonoscillatory second-order difference equation", J. Math. Anal. Appl. 255 (2001) 627-635.

[20] D. Willet and J. S. W. Wong, "On the discrete analogues of some generalizations of Gronwall's inequality", Monatsh. Math. 69 (1964) 362-367. 